\title{
Histogenesis of Kaposi's sarcoma in patients with and without acquired immune deficiency syndrome (AIDS)
}

\author{
R RUSSELL JONES, ${ }^{*} \dagger$ J SPAULL, ${ }^{*}$ C SPRY,† E WILSON JONES* \\ From the *St John's Hospital for Diseases of the Skin, London, and the $\uparrow$ Royal Postgraduate Medical School, \\ London
}

SUMMARY Immunohistochemical studies were performed in thirty skin biopsies from patients with Kaposi's sarcoma, who did and did not have the acquired immune deficiency syndrome (AIDS). Tumour histogenesis was rigorously tested using a battery of endothelial cell markers, which included two new monoclonal antibodies, EN4 and PAL E. These are both specific for endothelial cells and can be visualised in appropriately fixed paraffin embedded tissue. Whereas EN4 labels all $\mathrm{N}$ endothelial cells, PALE is negative in endothelium of lymphatic derivation. Lectin binding with $\mathrm{O}$ Ulex europaeus agglutinin 1 (UEA-1) and the presence of factor VIII related antigen (FVIIIRA) and laminin were also examined.

In nodular lesions of Kaposi's sarcoma the spindle cell areas were positive with EN4 and UEA-1, negative with PALE, and showed focal staining for FVIIIRA and laminin. These results confirm that the tumour is of endothelial cell origin. Six patch stage lesions showed a network of angulated spaces, lined by cells that were positive with EN4 and UEA-1, negative with PALE and antiFVIIIRA, and showed only weak staining for laminin. This pattern was observed in both AIDS and non-AIDS related cases and strongly favours a lymphatic derivation for the tumour. This has important implications as it suggests that lymphatic endothelium may have special characteristics that lead to neoplastic transformation in patients with retrovirus infection.

Establishing the histogenesis of Kaposi's sarcoma is complicated by the diverse clinical and histological patterns associated with the tumour. Historically, European cases have been regarded as a slowly evolving, with indolent tumour affecting elderly patients, usually of Eastern European or Mediterranean origin. ${ }^{1}$ It has long been recognised, however, that fatal forms of the tumour exist, particularly in Central Africa, ${ }^{23}$ and that the neoplasm may pursue a more aggressive form in patients who are immunosuppressed. ${ }^{4-6}$ The occurrence of Kaposi's sarcoma in patients with acquired immune deficiency syndrome (AIDS) has therefore changed our perception of this tumour and prompted research into its histogenesis.

Most previous studies of Kaposi's sarcoma have used the presence of factor VIII related antigen (FVIIIRA) to investigate tumour histogenesis. FVIIIRA, however, does not preserve well in formalin fixed tissue, background staining is a problem, and false positivity has been reported in tumours of non-vascular derivation. ${ }^{78}$ These factors may account for the conflicting reports obtained in pre-8 vious studies of Kaposi's sarcoma. ${ }^{9-13} \mathrm{~A}$ few authors $\overline{0}$ have used lectin binding with UEA-1, but again no consensus has emerged in studies of Kaposi's sar- 0 coma. ${ }^{1415}$ There is also the need to distinguish between vascular and lymphatic endothelium.

Although Ulex europaeus agglutinin 1 (UEA-1), \ FVIIIRA, and laminin have all been used to identify을 capillaries of vascular origin, ${ }^{16-18}$ these distinctions $N$ were not found in our studies of normal skin using cryostat sections. Lymphatic capillaries, for example, $\stackrel{\sim}{\circ}$ showed positive lectin binding and weak laminin ${ }_{\omega}$ staining in the periendothelial region. ${ }^{19}$ Furthermore, 0 FVIIIRA is present in the endothelium of larger lym-e phatic channels, ${ }^{1920}$ so its presence in nodules of $\frac{}{\Phi}$ Kaposi's sarcoma needs to be interpreted with cau-? tion.

This study used two new monoclonal antibodies, EN4 and PALE. Although both monoclonals are specific for endothelial cell they have different distri- $\stackrel{\mathbb{Q}}{\mathbb{Q}}$ butions in the normal cutaneous vasculature. Thus 
PALE, which recognises an antigen associated with pinocytic vesicles, stained all capillaries, venules, and small or medium sized veins, but it is negative in lymphatic endothelium and only weakly positive in arteriolar endothelium. ${ }^{21}$ By contrast, EN4 recognises a plasma membrane antigen present on all endothelial cells and therefore stains both vascular and lymphatic endothelium with vascular endothelium showing stronger reactivity than the lymphatic type. ${ }^{19}$

Virtually all previous studies of Kaposi's sarcoma have relied either on formalin fixation, which compromises antigen preservation, or frozen sections, which obscure anatomical detail and are biologically hazardous. To preserve labile endothelial cell antigens we used two methods of fixation-methacarn and periodate-lysine-paraformaldehyde (PLP)-before paraffin embedding. ${ }^{22}$ Although methacarn was superior to formalin for the immunohistochemical localisation of FVIIIRA, cold fixation in PLP was needed for the two monoclonal antibodies EN4 and PAL E. ${ }^{19}$ This combination of endothelial cell markers and fixation methods provided a unique opportunity for studying the histogenesis of Kaposi's sarcoma in AIDS and non-AIDS related cases.

\section{Material and methods}

\section{SAMPLES AND FIXATION}

Thirty skin biopsy specimens taken from 13 patients with Kaposi's sarcoma, three of whom had AIDS, were processed by one of two methods.

Methacarn fixation The tissue was fixed for 24 hours in a solution containing $60 \%$ methanol, $30 \%$ chloroform, and $10 \%$ glacial acetic acid before processing to paraffin wax via alcohol and xylene.

PLP fixation The tissue was fixed for two hours at $4^{\circ} \mathrm{C}$ in a solution containing $0.1 \mathrm{M} \mathrm{Nalo4}, 0.075 \mathrm{M}$ lysine, $2 \%$ freshly prepared paraformaldehyde in $0.0375 \mathrm{M}$ NaHPO4 buffer. The block was then rapidly vacuum embedded at $60^{\circ} \mathrm{C}$ after dehydrating with absolute ethanol and clearing in xylene at $4^{\circ} \mathrm{C}$.

\section{REAGENTS}

UEA-1 (Sigma) was made up at $0.002 \%$ in $0.5 \%$ bovine serum albumin.

Rabbit anti-UEA-1 (diluted 1/100 in phosphate buffered saline), rabbit anti-FVIIIRA (diluted 1/100 in phosphate buffered saline), peroxidase conjugated swine anti-rabbit immunoglobulin (diluted 1/125 in phosphate buffered saline), and peroxidase conjugated rabbit antimouse immunoglobulin (diluted 1/75 in phosphate buffered saline) were purchased from Dakopatts.

Rabbit antilaminin (diluted $1 / 100$ in phosphate buffered saline) was obtained from Bethesda. Mono- clonal anti-FVIIIRA was obtained from Dakopatts and used at a dilution of $1 / 75$ in phosphate buffered saline.

The monoclonal antibody EN4, which was raised by immunising mice with human umbilical cord endothelial cells and fusing the spleen cells with mouse NS1 cells, was produced and characterised as described previously. ${ }^{24}$ This antibody binds to all blood and lymphatic vessels in the skin. ${ }^{19}$ Monoclonal antibody PAL E binds to endothelial cell membrane antigens in blood vessels but not lymphatics. Both monoclonal antibodies were used undiluted.

Sections were stained by indirect immunoperoxidase staining. Endogenous peroxidase was blocked using methanol with $1 \% \mathrm{H}_{2} \mathrm{O}_{2}$. Binding of the peroxidase labelled second antibodies was shown using 3-3-diaminobenzidine and $\mathrm{H}_{2} \mathrm{O}_{2}$, and sections were counterstained with Mayer's haematoxylin. Methacarn and PLP fixed sections were digested with $1 \%$ protease solution (Sigma) for 15 minutes before staining with antibodies to laminin and antibody PALE. Sections treated with antibody EN4 and UEA-1 followed by anti-UEA-1 were not predigested. Monoclonal and polyclonal antisera to FVIIIRA were used with and without protease predigestion.

\section{Results}

\section{HISTOLOGY}

The nodular lesions of Kaposi's sarcoma comprise a variable mixture of vascular and spindle cell elements. The spindle cell areas contained a large number of slit like spaces, which were stuffed with erythrocytes and gave the tumour a loosely textured appearance. Sectioned transversely, these areas exhibited a sponge like or honeycomb network of blood filled spaces. Usually these spaces lacked a discernible row of lining cells. Local collections of capillary like vessels were, however, present within the body of the tumour, as well as larger vascular structures. These different elements need to be considered separately in establishing histogenesis. Our study included 18 biopsy specimens from nodular lesions, three of which were from AIDS related cases.

Early lesions of Kaposi's sarcoma showed a rather different histology. Small nodular foci were commonly seen as the earliest manifestation of a Kaposi lesion. These contained a few spindle cells and numerous vascular channels and were usually associated with an inflammatory cell infiltrate. In addition, biopsy specimens from several patch stage lesions were characterised by a network of empty angulated spaces within the dermal connective tissue, giving rise to a dissection of collagen appearance, not dissimilar to that seen with angiosarcoma. ${ }^{25}$ Because these spaces are lined by a flat row of cells, the term 
Table 1 Immunostaining of normal vessels in PLP fixed tissue

\begin{tabular}{|c|c|c|c|c|c|}
\hline & EN4 & $P A L-E$ & $F V I I I R A$ & Laminin & $U E A-I$ \\
\hline $\begin{array}{l}\text { Lymphatic capillaries } \\
\text { Larger valvular lymphatic nodes } \\
\text { Small and medium sized arteries } \\
\text { Arterioles } \\
\text { Blood capillaries } \\
\text { Venules } \\
\text { Small and medium sized veins }\end{array}$ & $\begin{array}{l}++ \\
++ \\
+++ \\
+++ \\
+++ \\
+++ \\
+++\end{array}$ & $\begin{array}{l}- \\
- \\
+1- \\
+1- \\
++ \\
++ \\
++\end{array}$ & $\begin{array}{l}- \\
+1- \\
++ \\
++ \\
+++ \\
+++ \\
++\end{array}$ & $\begin{array}{l}+1- \\
+ \\
++ \\
++ \\
++ \\
++ \\
++\end{array}$ & $\begin{array}{l}+ \\
++ \\
++ \\
++ \\
++ \\
++ \\
++\end{array}$ \\
\hline
\end{tabular}

+++ intense staining; ++ moderate staining; + weak staining; - negative result.

"lymphangiomatous" has been applied to this particular histological variant. ${ }^{26}$ Although not present in all patch stage lesions, it was particularly apparent in AIDS related cases. Our study included 12 biopsy specimens of patch stage lesions; six of these showed lymphangiomatous changes, and of these, three were from AIDS related cases.

\section{IMMUNOHISTOLOGY}

Immunoperoxidase labelling was graded in a semiquantitative fashion, according to the following scale: intense staining $(+++)$; moderate staining $(++)$; weak staining $(+)$, negative $(-)$. Blood and lymphatic vessels were identified in the normal skin surrounding Kaposi tumours. Table 1 lists their staining characteristics.

\section{NODULAR LESIONS}

Within the tumour nodules vessels could be identified that showed the same staining pattern and the same degree of reactivity as normal vessels in the surrounding dermis. Some of these vessels labelled with all five markers and presumably represent the normal vascular supply to the tumour. Other structures with dilated or irregular lumina showed weaker staining reactions than normal endothelial cells and were negative with anti-FVIIIRA and PALE. These vessels could either represent dilated lymphatics or part of the proliferative process.

In nodular lesions of Kaposi's sarcoma the only marker that was consistently positive in the spindle cell areas was EN4, which showed weak or moderate reactivity in all tumours examined (Fig. 1). PALE was consistently negative, and the other three markers were more variable in their expression. $\overrightarrow{\vec{\omega}}$ (Table 2). Thus most of the tumours showed weak to moderate lectin binding, but the degree of reactivity varied between different parts of the same tumour, $\omega$ and in some areas was entirely negative. Results obtained with the polyclonal antisera were more $\stackrel{\vec{N}}{\mathrm{~N}}$ difficult to interpret as non-specific staining can occur with polyclonal antisera and protease predigestion. $\stackrel{ }{J}$ This was particularly true of FVIIIRA, which is a $\vec{c}$ cytoplasmic marker and showed diffusion of the reaction product around principal blood vessels. Serial sections were therefore examined using both mono- $\infty$ clonal and polyclonal anti-FVIIIRA reagents-with and without protease predigestion. These studies showed focal areas of moderate reactivity for FVIIIRA within the tumour nodules. These areas corresponded to foci of well differentiated capillary proliferation, which histologically were difficult to $\stackrel{\mathbb{\Omega}}{\complement}$ distinguish from the surrounding tumour mass. The $\overrightarrow{\vec{B}}$ areas of undifferentiated spindle cell proliferation $\frac{3}{3}$ were either negative or only weakly positive with the monoclonal antibody to FVIIIRA and the polyclonal antisera to laminin (Table 2).

\section{EARLY LESIONS}

Lesions showing small prolifative foci showed a rather different staining pattern to the larger nodular $\frac{9}{3}$ lesions. In several cases there was moderate staining for FVIIIRA and laminin, as well as variable PALE reactivity and strong reactivity with EN4 and UEA-1 $\frac{7}{0}$ (Fig. 2). These early lesions were, however, highly vascular, and care was needed to distinguish the $\mathcal{N}$ abnormal spindle cells from proliferating vessels with strongly stained endothelium.

Table 2 Nodular lesions and immunostaining pattern of spindle cell component in nine methacarn and nine PLP fixed skin biopsy specimens

\begin{tabular}{|c|c|c|c|c|c|}
\hline $\begin{array}{l}\text { Degree of } \\
\text { reactivity }\end{array}$ & $F V I I I R A$ & $U E A-I$ & Laminin & $P A L E$ & $E N 4$ \\
\hline $\begin{array}{l}\text { Moderate } \\
\text { Weak } \\
\text { Negative }\end{array}$ & $\begin{array}{r}0 \\
2 \\
16\end{array}$ & $\begin{array}{l}9 \\
6 \\
3\end{array}$ & $\begin{array}{r}0 \\
4 \\
14\end{array}$ & $\begin{array}{l}0 \\
0 \\
7\end{array}$ & $\begin{array}{l}7 \\
2 \\
0\end{array}$ \\
\hline
\end{tabular}




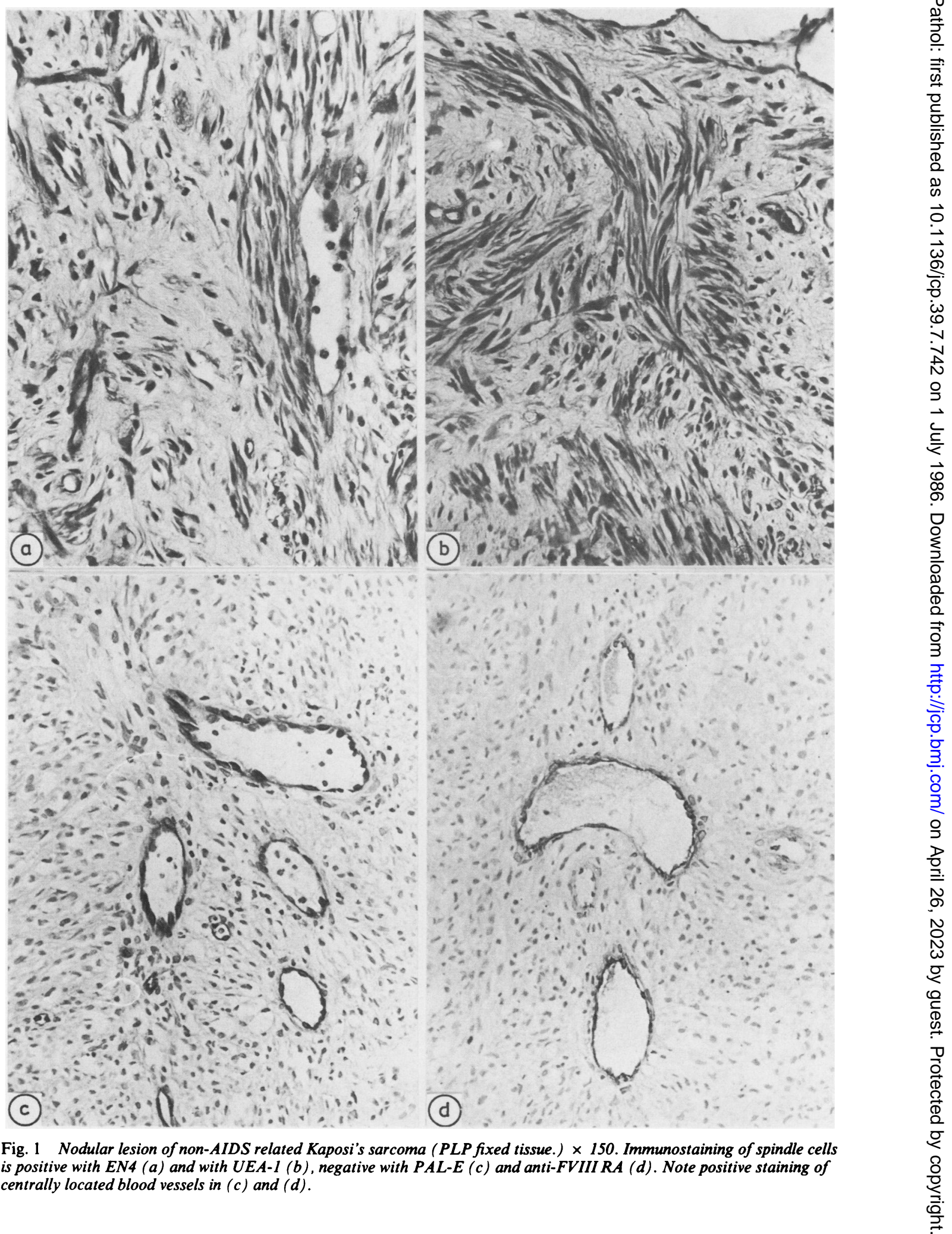




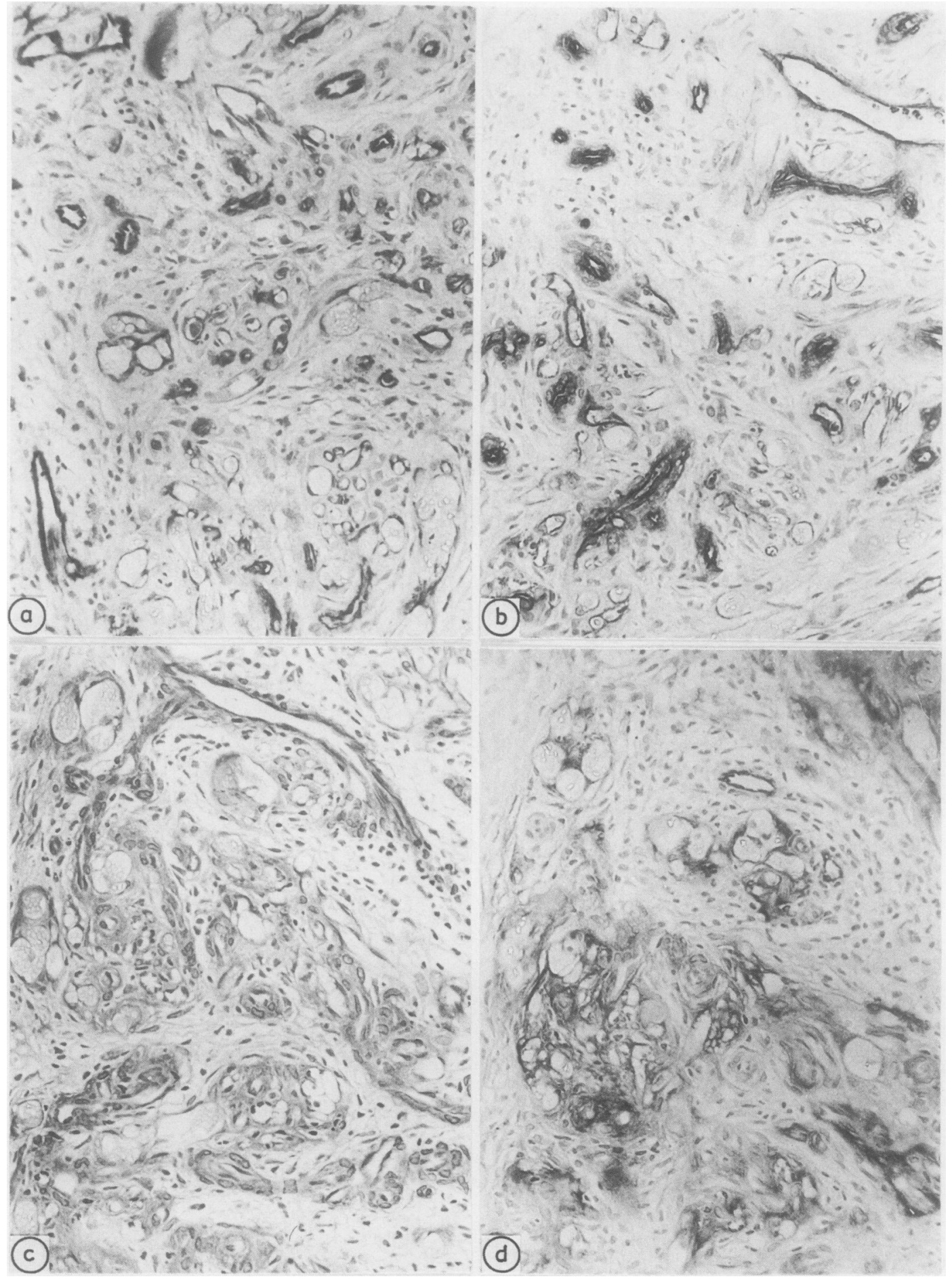

Fig. 2 Immunostaining of proliferating vessels in an early lesion on non-AIDS related Kaposi's sarcoma. (PLP fixed tissue.) $\times 150$. (a) monoclonal antibody EN4; (b) lectin binding-UEA-1; (c) polyclonal laminin antisera;

(d) monoclonal anti FVIII RA. Note slight diffusion of reaction product around positively stained blood vessel endothelium (monoclonal anti-FVIII RA: protease predigestion).
告

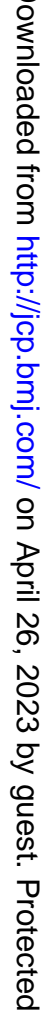




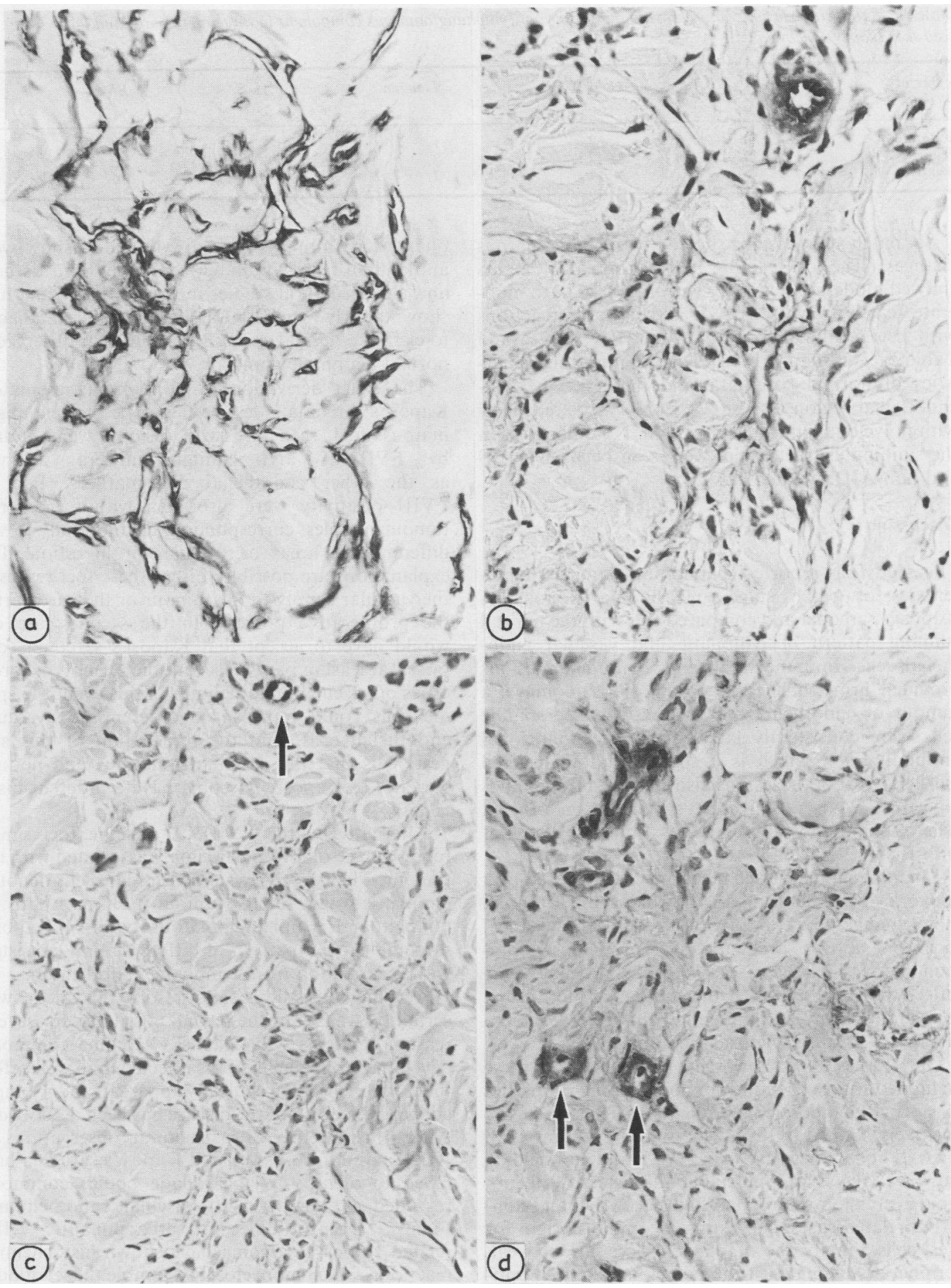

Fig. 3 Immunostaining of lining cells from lymphangiomatous variant of AIDS related Kaposi's sarcoma. Serial sections of PLP fixed tissue $(\times 350)$ show moderate reactivity with EN4 $(a)$, weak lectin binding with UEA-1 (b), and negative reactions with PALE (c), and monoclonal anti FVIII RA (d). Arrows indicate positive staining in adjacent blood vessels. 
Table 3 Patch stage lesions: immunostaining pattern of lymphangiomatous component in three methacarn and three PLP fixed skin biopsy specimens

\begin{tabular}{llllll}
\hline $\begin{array}{l}\text { Degree of } \\
\text { reactivity }\end{array}$ & FVIII RA & UEA-I & Laminin & PALE \\
\hline Moderate & 0 & 3 & 1 & 3 & 0 \\
Weak & 0 & 2 & 2 & 0 & 3 \\
Negative & 6 & 1 & 1 & 3 \\
\hline
\end{tabular}

Six patch stage lesions showed lymphangiomatous changes. The cells lining these angulated spaces showed moderate reactivity with EN4, weak to moderate reactivity with UEA-1, and negative reactions with PALE and monoclonal FVIIIRA (Fig. 3 and Table 3). This mimics precisely the staining characteristics of normal lymphatic endothelium. The subendothelial region also showed only weak reactivity with polyclonal antisera to laminin. No differences in the staining characteristics were seen between AIDS and non-AIDS related cases.

\section{Discussion}

This study has reported the immunostaining characteristics of patch stage and nodular lesions of Kaposi's sarcoma and compared this with the normal staining pattern of lymphatic and vascular endothelial cells. Immunostaining of 18 nodular lesions provided firm evidence that Kaposi's sarcoma is a tumour of endothelial cell origin. Thus the spindle cells were consistently labelled by the monoclonal antibody EN4, which is a specific endothelial cell marker. Most of the lesions also exhibited lectin binding with UEA-1, but this is not specific for endothelial cells, and immunostaining was less constant than that with EN4.

Lymphangiomatous changes are characterised by a network of proliferating angulated spaces. Although these changes have been described in the classical form of Kaposi's sarcoma, ${ }^{26}$ it is particularly common in AIDS related cases. While this histology may reflect the stage at which the tumour is biopsied, it seems more likely that patients with AIDS develop a distinctive form of Kaposi's sarcoma.

Our studies have shown that the cells lining these angulated spaces have the immunostaining properties of normal lymphatic endothelium. Thus they are positively stained by EN4, show lectin binding with UEA-1, but are negative with PAL E and monoclonal anti-FVIIIRA. No appreciable differences of phenotypic expression were found between AIDS and nonAIDS related cases, suggesting a common origin for these cells. Finally, the angulated spaces showed weak peripheral staining with the polyclonal laminin antisera. Again, however, this does not exclude a lym- phatic derivation, as normal lymphatics show weak $\vec{\circ}$ antilaminin reactivity in appropriately fixed tissue, ${ }^{19} \overrightarrow{\vec{\omega}}$ and ultrastructural studies in Kaposi's sarcoma have $\stackrel{\sigma}{\omega}$ shown poorly formed basal lamina material adjacent $\overline{ }$ to cleft like dermal spaces similar to that observed in normal lymphatic glands. ${ }^{27}$

The exact derivation of non-lymphangiomatous $-v$ Kaposi's sarcoma is less certain. The discrete angio- $\vec{N}$ matous foci seen in early lesions were stained 0 by FVIIIRA and laminin antisera, as well as the other endothelial cell markers. Foci of $c$ FVIII positivity were also seen within the larger tumour nodules, corresponding histologically to well $\vec{\bullet}$ differentiated areas of vascular proliferation. Two explanations are possible. Either these foci represent the vascular supply to the tumour or they are part of the proliferative process. In the second case one would have to accept that Kaposi's sarcoma comprises, at least in part, cells with the phenotypic prop- $\frac{\mathscr{Q}}{\not}$ erties of vascular endothelium. In this event the spin- $\propto$ dle cells could represent undifferentiated vascular $\overrightarrow{\vec{O}}$ endothelial cells, and the implication of this sug- 3 gestion is that the lymphangiomatous and nodular varieties of Kaposi's sarcoma have a different histogenesis.

Alternatively, the FVIIIRA positive foci simply: represent the rich vascular supply associated with this 3 . tumour. The negatively stained spindle cells denote ao lymphatic origin for the tumour itself, and both the nodular and lymphangiomatous types of Kaposi's sarcoma show a pattern of differentiation consistent with a derivation from lymphatic endothelium.

Although our study cannot resolve this dilema with certainty, we favour the second possibility for severalos reasons. Patients with Kaposi's sarcoma commonly N develop lymphoedema of the affected limb well before N the tumours become clinically apparent, and a lymph- $\omega$ adenopathic form of the disease has been described in African children. ${ }^{2}$ Ultrastructural observations of AIDS and non-AIDS related Kaposi's sarcoma have failed to identify Weibel-Palade bodies commonly regarded as a marker for vascular endothelium. ${ }^{27} \frac{0}{\circ}$ Finally, recent studies of predominantly AIDS related Kaposi's sarcoma have shown histochemical reactions, which more closely resemble those found in 0 normal lymphatic endothelium. Thus 5-nucleotidase? 
was positive in five of sixteen spindle cell lesions, whereas adenosine triphosphatase and alkaline phosphatase activity were negative. ${ }^{28}$

In conclusion, we found that Kaposi's sarcoma is certainly a tumour of endothelial cell origin. A derivation from lymphatic endothelium seemed certain for the lymphangiomatous variant and probable for the nodular variety of the tumour. EN4 has proved to be a reliable marker for malignant endothelial cells and should be of value in diagnosis. Further work is required to establish whether retroviruses selectively colonise lymphatic endothelium and whether lymphatic endothelium cells might be able to support the growth of AIDS related retroviruses.

We are grateful to Dr DJ Ruiter from the University Medical Centre, Leiden, for supplying the monoclonal antibody PAL E. We thank Dr Neil Smith and other colleagues who allowed us to study patients under their care, or who provided biopsy material.

\section{References}

${ }^{1}$ Cox FH, Helwig EB. Kaposi's sarcoma. Cancer 1959;12:289-98.

${ }^{2}$ Slavin G, Cameron HM, Forbes C, Morton-Mitchell R. Kaposi's sarcoma in east African children. A report of 51 cases. J Pathol 1970;100:187-99.

${ }^{3}$ Templeton AC. Studies in Kaposi's sarcoma. Cancer 1972; 30:854-67.

${ }^{4}$ Gange RW, Wilson Jones E. Kaposi's sarcoma and immunosuppressive therapy: an appraisal. Clin Exp Dermatol 1978;3:135-46.

${ }^{5}$ Harwood AR, Osoba D, Hofstader SL, et al. Kaposi's sarcoma in recipients of renal transplants. Am J Med 1979;67:759-65.

${ }^{6}$ Hoshaw RA, Schwartz RA. Kaposi's sarcoma after immunosuppressive therapy with prednisone. Arch Dermatol 1980;116:1280-82.

${ }^{7}$ Millard PR, Heryet AR. An immunohistochemical study of factor VIII related antigen and Kaposi's sarcoma using polyclonal and monoclonal antibodies. J Pathol 1985;146:31-8.

${ }^{8}$ Wilson AJ. Factor VIII related antigen staining by immunoperoxidase technique in smaller laboratories: a potential problem. Am J Clin Pathol 1984;81:117-20.

${ }^{9}$ Sehested M, Hou-Jensen K. Factor VIII related antigen as an endothelial cell marker in benign and malignant diseases. Pathol Anat 1981;391:217-25.

${ }^{10}$ Burgdorf W, Mukai K, Rosai J. Immunohistochemical identification of factor VIII related antigen in endothelial cells of cutaneous lesions of alleged vascular nature. Am J Clin Pathol 1981;75:167-71.

${ }^{11}$ Guarda LG, Silva EG, Ordanez NG, Smith JL, Jr. Factor VIII in Kaposi's sarcoma. Am J Clin Pathol 1981;76:197-200.

${ }^{12}$ Modlin RL, Hofman FM, Kemp FRA, Taylor CR, Conant MA,
Rea TH. Kaposi's sarcoma in homosexual men: an immunohistochemical study. J Am Acad Dermatol 19??;8:620-27.

${ }^{13}$ Nadjii M, Mirales AR, Ziegles-Weismann J, Penneys NS. Kaposi's sarcoma: immunohistologic evidence for an endothelial origin. Arch Pathol Lab Med 1981;105:274-5.

${ }^{14}$ Miettinen M, Holthofer H, Lehto VP, Miettinen A, Virtanen I. Am J Clin Pathol 1983;79:32-6.

${ }^{15}$ Ordonez NG, Batsakis JG. Comparison of Ulex europaeus 1 lectin and factor VIII-related antigen in vascular lesions. Arch Pathol Lab Med 1984;108:129-32.

${ }^{16}$ Mortimer PS, Cherry GW, Jones RL, Barnhill RL, Ryan TJ. The importance of elastic fibres in skin lymphatics. $\mathrm{Br} J$ Dermatol 1983;108:561-6.

${ }^{17}$ Walker RA. Ulex europaeus 1-peroxidase on a marker of vascular endothelium: its application in routine histopathology. J Pathol 1985;146:123-7.

${ }^{18}$ Barsky SH, Baker A, Siedgal GP, Togo S, Liotta LA. Use of antibasement membrane antibodies to distinguish blood vessel capillaries from lymphatic capillaries. Am J Surg Pathol 1983;7:667-77.

${ }^{19}$ Holden CA, Spaull J, Williams R, Spry CJF, Russell Jones R, Wilson Jones $E$. The distribution of endothelial cell antigens in cutaneous tissue using methacarn and periodate lysine paraformaldehyde fixation. $J$ Immunol Methods (in press).

${ }^{20}$ Svanholm H, Nielsen K, Hauge P. Factor VIII-related antigen and lymphatic collecting vessels. Arch Pathol Anat 1984;404:223-8.

${ }^{21}$ Schlingemann RO, Dingjan GM, Emeis JJ, Blok J, Warnaar SO, Ruiter DJ. Monoclonal antibody PAL-E specific for endothelium. Lab Invest 1985;52:71-6.

${ }^{22}$ Mitchell D, Ibrahim S, Gusterton BA. Improved immunohistochemical localisation of tissue antigens using modified methacarn fixation. J Histochem Cytochem 1985;33:491-5.

${ }^{23}$ Collings LA, Poulter LW, Janossy G. The demonstration of cell surface antigens on $\mathrm{T}$ cells, $\mathrm{B}$ cells and accessory cells in paraffin embedded human tissues. J Immunol Methods 1984;75:227-39.

${ }^{24}$ Cui YC, Tai P-C, Gatter KC, Mason DY, Spry CJF. A vascular endothelial cell antigen with restricted distribution in human foetal, adult and malignant tissues. Immunology 1983;49:183-9.

${ }^{25}$ Wilson Jones E. Malignant vascular tumours. Clin Exp Dermatol 1976;1:287-311.

${ }^{26}$ Gange RW, Wilson Jones E. Lymphangioma-like Kaposi's sarcoma. Br J Dermatol 1979;100:327-34.

${ }^{27}$ McNutt NS, Fletcher V; Conant MA. Early lesions of Kaposi's sarcoma in homosexual men: an ultrastructural comparison with other vascular proliferations in skin. Am $J$ Pathol 1983;111:62-77.

${ }^{28}$ Beckstead JH, Wood GS, Fletcher V. Evidence for the origin of Kaposi's sarcoma from lymphatic endothelium. Am J Pathol (in press).

Requests for reprints to: Dr R Russell Jones, St John's Hospital for Diseases of the Skin, 5 Lisle Street, Leicester Square, London WC2H 7BJ, England. 